\title{
Die fachtechnisch verantwortliche Person im Verwaltungsstrafverfahren von Swissmedic
}

\author{
Ines Meier *
}

Jedes im Handel oder der Herstellung von Arzneimitteln tätige Pharmaunternehmen in der Schweiz verfügt über eine «fachtechnisch verantwortliche Person». Diese ist nicht nur die betriebsinterne Anlaufstelle für heilmittelspezifische Fragen rund um den Vertrieb oder die Herstellung von Arzneimitteln, sondern sie ist auch die primäre Ansprechperson für Swissmedic als Aufsichtsbehörde über die Heilmittel. Der folgende Beitrag beleuchtet die Rolle der fachtechnisch verantwortlichen Person in einem von Swissmedic geführten Verwaltungsstrafverfahren. Es zeigt sich, dass die Position der fachtechnisch verantwortlichen Person heilmittelstrafrechtlich stark exponiert ist.

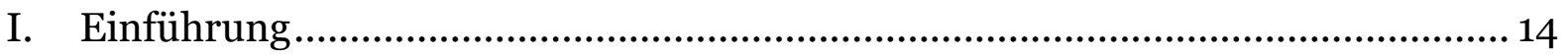

II. Tätigkeiten des Schweizerischen Heilmittelinstituts Swissmedic ....................... 14

1. Verwaltungsrechtliche Aufsicht über Heilmittel ........................................ 14

2. Strafverfolgung im Heilmittelbereich .................................................... 16

III. Die fachtechnisch verantwortliche Person ...................................................... 18

1. Tätigkeitsbereich der fachtechnisch verantwortlichen Person ....................... 18

2. Ausbildung und Vertrauenswürdigkeit................................................... 19

3. Weisungsbefugnis und Unabhängigkeit ................................................20

4. Präsenzzeiten und Stellvertretung .......................................................... 21

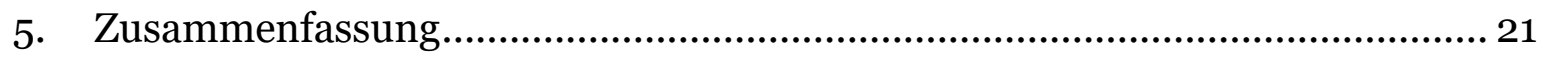

IV. Strafrechtliche Verantwortlichkeit der fachtechnisch verantwortlichen Person .22

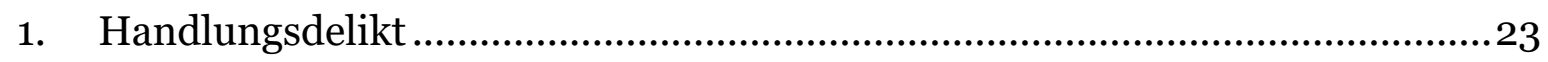

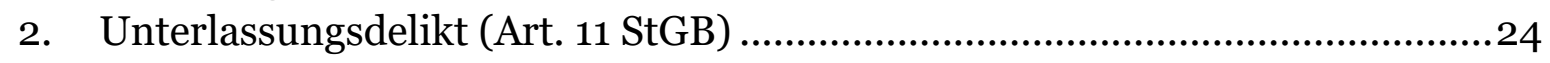

3. Geschäftsherrenhaftung (Art. 6 Abs. 2 und 3 VStrR) ...................................25

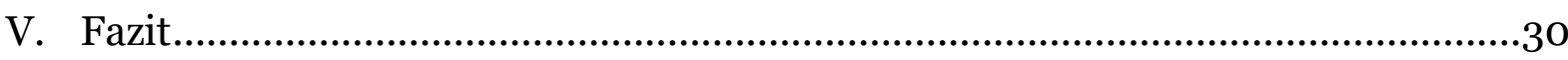

Zitiervorschlag: Ines Meier, Die fachtechnisch verantwortliche Person im Verwaltungsstrafverfahren von Swissmedic, in: sui-generis 2017, S. 13

URL: $\quad$ sui-generis.ch/30

DOI: $\quad$ https://doi.org/10.21257/sg.30

* Ines Meier, Rechtsanwältin, Untersuchungsleiterin in der Abteilung Strafrecht von Swissmedic. Die Ausführungen in diesem Aufsatz geben ausschliesslich die persönliche Auffassung der Autorin wieder. Mein herzlicher Dank gebührt Fürsprecher Michael Burri, RA Dr. iur. Andrea Schütz, LL.M., und RA Dr. iur. Séverine Lachat-Boillat für die kritische Durchsicht des Manuskripts und die wertvollen Hinweise und Anregungen.

Dieses Werk ist lizenziert unter einer Creative Commons Namensnennung - Weitergabe unter gleichen Bedingungen 4.0 International Lizenz. 


\section{Einführung}

1 In der Schweiz verfügen aktuell über 1000 Unternehmen über eine Betriebsbewilligung vom Schweizerischen Heilmittelinstitut Swissmedic ${ }^{1}$. Zahlreiche dieser Betriebe sind im Grosshandel tätig, das heisst sie kaufen Arzneimittel ein und verkaufen diese innerhalb der Schweiz an andere Unternehmen, nicht jedoch an den Endverbraucher, weiter. Um eine Betriebsbewilligung von Swissmedic zu erhalten, muss jedes Unternehmen, das Arzneimittel herstellt oder damit handelt, über eine sog. «fachtechnisch verantwortliche Person» verfügen. Diese ist für die Einhaltung der heilmittelrechtlichen Vorschriften bei der Herstellung von Arzneimitteln und deren Vertrieb verantwortlich. Ihr kommen damit innerhalb des jeweiligen Betriebs nicht nur weitreichende Befugnisse zu, sondern es obliegt ihr auch eine umfassende Verantwortung mit Blick auf die Heilmittelsicherheit. Die fachtechnisch verantwortliche Person ist erste Ansprechperson für sämtliche Belange und Fragen der Heilmittelsicherheit, mögen diese von Angestellten oder Vorgesetzten des Unternehmens, von den Aufsichtsbehörden oder von anderen Pharmafirmen kommen. Sie stellt - auf den Punkt gebracht - das heilmittelrechtliche Rückgrat eines jeden im Pharmabereich tätigen Unternehmens dar².

Auf der Webseite von Swissmedic findet sich eine öffentlich zugängliche Liste, auf welcher sämtliche Bewilligungsinhaber aufgeführt sind: www.swissmedic.ch/bewilligungen/00155/0024/ 00253.

2 Vgl. auch Anaï Cordoba, Produits thérapeutiques défectueux et résponsabilités pénales, in: Jusletter vom 28.1.2013, Rz 85, wonach «le responsable technique [est] un élément clé dans une firme pharmaceutique».
2 Auf den fachtechnisch Verantwortlichen richtet sich der Fokus in der Regel auch dann, wenn die Abteilung Strafrecht von Swissmedic ein Verwaltungsstrafverfahren wegen Verdachts auf Widerhandlungen gegen das Heilmittelgesetz und seine Ausführungsverordnungen durch eine Pharmafirma eröffnet hat. Dabei zeigt sich, dass die fachtechnisch verantwortliche Person - wenn denn eine solche überhaupt bezeichnet wurde häufig auch verurteilt wird, sofern nicht das Unternehmen selbst gebüsst wird. Dieser Beitrag zeigt die Gründe dafür auf.

3 Zuerst folgt ein Überblick über die Tätigkeiten des Schweizerischen Heilmittelinstituts Swissmedic (II.) Anschliessend werden die Aufgaben der fachtechnisch verantwortlichen Person beleuchtet (III.), bevor letztlich der Frage nachgegangen wird, wie es sich mit ihrer strafrechtlichen Verantwortlichkeit verhält (IV.). Ein Fazit rundet die Ausführungen $\mathrm{ab}(\mathrm{V}$.$) .$

\section{Tätigkeiten des Schweizerischen Heilmittelinstituts Swissmedic}

\section{Verwaltungsrechtliche Aufsicht über Heilmittel}

4 Der Bund erliess gestützt auf Art. 118 Abs. 2 BV3 das Heilmittelgesetz 4 . Mit dem Vollzug beauftragte er das Schweizerische Heilmittelinstitut Swissmedic (Art. 82 Abs. 1 HMG). Dieses hat als Gesundheitspolizeibehörde dafür zu sorgen,

$3 \longdiv { \text { Bundesverfassung der Schweizerischen Eidge- } }$ nossenschaft vom 18. April 1999 (BV; SR 101).

4 Bundesgesetz über Arzneimittel und Medizinprodukte vom 15. Dezember 2000 (HMG; SR 812.21). 
dass zum Schutz der Gesundheit von Mensch und Tier nur qualitativ hoch stehende, sichere und wirksame Heilmittel (Arzneimittel und Medizinprodukte5) in Verkehr gebracht werden (Art. 1 Abs. 1 HMG).

5 Um den Gesetzeszweck zu erfüllen, erfuhr der Heilmittelmarkt eine starke Regulierung durch den Gesetzgeber. Entsprechend ist eine Tätigkeit im Zusammenhang mit Arzneimitteln ohne kantonale oder Swissmedic-Bewilligung nicht zulässig6. Swissmedic erteilt Betriebsbewilligungen an Unternehmen, die Arzneimittel in industriellem Ausmass herstellen (sog. Herstellungsbewilligung7), oder damit handeln wollen ${ }^{8}$ (Bewilligung für die Ausfuhr'9, die Einfuhr ${ }^{10}$, den

Art. 2 Abs. 1 lit. a HMG. Vgl. zu den Definitionen Art. 4 Abs. 1 lit. a und b HMG.

6 Im Gegensatz zu den Arzneimitteln durchlaufen die Medizinprodukte kein Zulassungsverfahren. Für diese Produkte hat die Schweiz auf der Basis von bilateralen Verträgen das System der Konformitätsbewertung bzw. Zertifizierung der Europäischen Union übernommen. Die Konformität zu den europäischen Medizinprodukte-Bestimmungen (Richtlinien, harmonisierte Normen etc.) wird dabei - anders als bei Arzneimitteln - durch den Hersteller selber bestätigt oder von privaten Stellen bewertet. Medizinprodukte höherer Risikoklassen dürfen von einem Hersteller oder Importeur erst in Verkehr gebracht werden, wenn das jeweilige Konformitätsbewertungsverfahren erfolgreich abgeschlossen wurde (Art. 46 Abs. 1 HMG; Art. 4 ff. der Medizinprodukteverordnung vom 17. Oktober 2001 [MepV; SR 812.213]). Die Konformitätsbewertungsstellen führen eine Überprüfung der Medizinprodukte durch und bescheinigen durch Anbringen der «CE»-Kennzeichnung, dass es den europäischen Medizinprodukte-Bestimmungen entspricht.

7 Art. 5 Abs. 1 HMG; zu den spezifischen Anforderungen vgl. Art. 3 ff. der Arzneimittel-Bewilligungsverordnung (AMBV; SR 812.212.1).

8 Gemeint ist die «Vermittlung: das Beziehen, Importieren, Exportieren, Aufbewahren, Lagern, Anbieten, Anpreisen, entgeltliche oder unentgeltliche Übertragen oder Überlassen von Arzneimitteln einschliesslich der Auslieferung, jedoch ohne die Abgabe» (Art. 2 lit. k AMBV).

9 Vgl. zu den spezifischen Anforderungen Art. 18
Grosshandel ${ }^{11}$ sowie für den Handel im Ausland ${ }^{12}{ }^{13}$. Die Bewilligungen werden erteilt, wenn die erforderlichen fachlichen und betrieblichen Voraussetzungen erfüllt sind und ein geeignetes Qualitätssicherungssystem vorhanden ist ${ }^{14}$, wozu auch die Benennung einer fachtechnisch verantwortlichen Person gehört. Aus-

Abs. 1 lit. b HMG, Art. 19 HMG, Art. 21 f. HMG sowie Art. 7 Abs. 2 AMBV.

10 Vgl. zu den spezifischen Anforderungen Art. 18 Abs. 1 lit. a HMG, Art. 19 f. HMG sowie Art. 7 ff. AMBV.

11 Vgl. zu den spezifischen Anforderungen Art. 28 f. HMG sowie Art. 7 Abs. 2 AMBV. Der Gesetzgeber versteht unter Grosshandel die «Vermittlung von Arzneimitteln an Personen, die ermächtigt sind, mit ihnen zu handeln, sie zu verarbeiten, abzugeben oder berufsmässig anzuwenden» (Art. 2 lit. e AMBV).

12 Vgl. Art. 18 Abs. 1 lit. c HMG, Art. 19 HMG, Art. 21 HMG sowie Art. 12 ff. AMBV.

13 Michael Burri, Swissmedic als Verwaltungsstrafbehörde im «Sandwich» zwischen Verwaltungsverfahren und kantonaler Strafverfolgung: Herausforderungen, Schnittstellen, Zielkonflikte, in: Aktuelle Herausforderungen für die Praxis im Verwaltungsstrafverfahren, hrsg. von Andreas Eicker, Bern 2013, S. 91-139, S. 92 f.; Catherine Manigley/Jean-Christophe Meroz/Matthias Stacchetti, La lutte contre les contrefaçons de produits pharmaceutiques, in: La lutte contre la contrefaçon et la criminalité pharmaceutique en Suisse, hrsg. von Isabelle Augsburger-Bucheli, Genf/Zürich/Basel 2010, S. 55-66, S. 61; Boris Kreit, Bekämpfung der Heilmittelkriminalität, Leitfaden für die Praxis, Bern 2016, S. 16; zum Ganzen: Swissmedic Merkblatt Bewilligungen. Zudem erteilt sie Bewilligungen für die Entnahme von Blut (Art. 34 f HMG, Art. 15 ff. AMBV). Darüber hinaus unterstehen weitere Tätigkeiten im Einzelfall einer Bewilligungspflicht, siehe z.B. Art. 32 und Art. 36 Abs. 2 AMBV; zur Bewilligungspflicht im Zusammenhang mit klinischen Versuchen mit Heilmitteln siehe Art. 53 f. HMG; Swissmedic Merkblatt Bewilligungen, S. 2 f.

14 Art. 6 Abs. 1 HMG (Herstellung); Art. 19 Abs. 1 HMG (Einfuhr, Ausfuhr und Handel im Ausland); Art. 28 Abs. 2 HMG (Grosshandel); Art. 34 Abs. 2 HMG (Entnahme von Blut und Blutprodukten); Art. 27 Abs. 1 AMBV; Ursula Eggenberger Stöckli, Gesundheitsrecht: Heilmittel, in: hrsg. von Giovanni Biaggini/Isabelle Häner/Urs Saxer/ Markus Schott (Hrsg.), Fachhandbuch Verwaltungsrecht, Zürich/Basel/Genf 2015, S. 573-626, N 15.69; vgl. auch BGE 131 II 44 E. 2.2. 
nahmen von der Bewilligungspflicht durch Swissmedic gibt es insofern, als die Herstellung von Arzneimitteln durch den Bundesrat einer kantonalen Bewilligungspflicht unterstellt (Herstellung von Arzneimitteln in kleinen Mengen für einen bestimmten Personenkreis, z.B. die Kundschaft einer Apotheke ${ }^{15}$ ) oder ganz von einer Bewilligungspflicht ${ }^{16}$ abgesehen werden kann ${ }^{17}$. Auch die Abgabe von Arzneimitteln an die Endabnehmer wie Apotheken, Drogerien, Spitäler und selbstdispensierende Ärzte unterliegt der kantonalen Bewilligungspflicht ${ }^{18}$.

\section{Strafverfolgung im Heilmittelbe- reich}

6 Mit dem Inkrafttreten des Heilmittelgesetzes per 1. Januar 2002 wurde der Bund, namentlich Swissmedic, hauptsächlich zuständig für die Strafverfolgung im Heilmittelbereich (vgl. Art. 90 Abs. 1 HMG). Damit stellt Swissmedic eine von wenigen Bundesverwaltungsbehörden dar, denen nebst ihrer aufsichtsrechtlichen Tätigkeit die Aufgabe und Kompetenz zur Strafverfolgung zukommen ${ }^{19}$. Innerhalb des Bereichs Rechts

15 Art. 5 Abs. 2 lit. a HMG.

16 Art. 5 Abs. 2 lit. b HMG.

17 Vgl. dazu auch Paul Richli, Regelungsschwerpunkte des Heilmittelrechts, Unter besonderer Berücksichtigung formeller Rechtsfragen, in: Das neue Heilmittelgesetz, Eine erste Bilanz, Referate der Tagung vom 19. November 2003 in Bern, hrsg. von Thomas Eichenberger/Tomas Poledna, Zürich/Basel/Genf 2004, S. 47-77, S. 55 f.; Burri (Fn. 13), S. 93 .

18 Vgl. Art. 4 Abs. 1 lit. f i.V.m. Art. 30 HMG; Burri (Fn. 13), S. 93; Eggenberger Stöckli (Fn. 14), $\mathrm{N} 15.45$.

19 Balsiger Betts, S. 53; Botschaft HMG, S. 3566. Die gleichen Kompetenzen kommen z.B. auch der Eidgenössischen Spielbankenkommission (vgl. Art. 57 Abs. 1 des Bundesgesetzes über Glücksspiele und Spielbanken [Spielbankengesetz]; SBG; SR 935.52) und der Eidgenössischen Zollverwaltung (vgl. Art. 128 Abs. 2 des Zollgesetzes; von Swissmedic obliegt die Strafverfolgung der dafür spezialisierten Abteilung Strafrecht ${ }^{20}$. Daneben zeichnen jedoch auch die Kantone für die Strafverfolgung verantwortlich ${ }^{21}$. Die jeweiligen Zuständigkeiten ergeben sich aus den entsprechenden Kompetenzen von Bund und Kantonen im Bereich der Eingriffs- bzw. Leistungsverwaltung22. Dabei ist jeweils gestützt auf die konkreten Normen des Heilmittelgesetzes und seiner Ausführungsverordnungen $\mathrm{zu}$ bestimmen, ob Swissmedic oder die Kantone für den verwaltungsrechtlichen Vollzug und damit auch für die Strafverfolgung zuständig sind23: Swissmedic ist für die Strafverfolgung verantwortlich, wenn es um die Herstellung von Arzneimitteln oder um die Einfuhr, die Ausfuhr oder den

ZG; SR 631.0) zu.

20 Die Abteilung Strafrecht hat die Arbeit am 1.1.2004 aufgenommen. Weiterführend zum Ganzen Séverine Lachat-Boillat/Matthias Stacchetti, La Lutte contre la criminalité pharmaceutique: état des lieux et perspectives, in: Réflexions romandes en droit de la santé, hrsg. von Anne-Sylvie Dupont/Olivier Guillod, Zürich/St. Gallen 2016, S. 211 ff., 213 f.; vgl. auch das Organigramm von Swissmedic, abrufbar unter www.swissmedic.ch/ueber/o0134/00441/ $00442 / 00444 / 00446$.

21 Vgl. Art. 90 Abs. 2 HMG.

22 Vgl. Art. 82 f. HMG; "Swissmedic ist zuständig bei der Herstellung von Arzneimitteln als "Massengüter" in Fabriken sowie beim Grosshandel und grenzüberschreitenden Verkehr mit Arzneimitteln, die Kantone bei deren Herstellung "nach Mass" in Apotheken und Spitälern sowie bei der Abgabe und Anwendung von Heilmitteln » (Burri (Fn. 13), S. 95).

23 Kreit, S. 42; Matthias Stachhetti, Les moyens pénaux tirés de la LPTh et particularités du droit pénal administratif, in: La lutte contre la contrefaçon et la criminalité pharmaceutique en Suisse, hrsg. von Isabelle Augsburger-Bucheli, Genf/Zürich/Basel 2010, S. 67-77, S. 70 f. Vgl. die detaillierte Aufzählung von Richli, in: Basler Kommentar [BSK] Heilmittelgesetz, hrsg. von Thomas Eichenberger/Urs Jaisli/Paul Richli, Basel 2006, Art. 82 N 9-11 sowie Art. 83 N 3, wo die einzelnen Bundes- und kantonalen Kompetenzen in der Eingriffs- und Leistungsverwaltung aufgelistet werden. 
Handel im Ausland sowie um den Grosshandel mit Arzneimitteln geht; werden Heilmittel unzulässigerweise an Konsumenten bzw. Patienten abgegeben, sind die kantonalen Staatsanwaltschaften zuständig24.

7 Die Strafbestimmungen sind im 8. Kapitel des Heilmittelgesetzes in den Art. 8690 HMG geregelt. Die Widerhandlungen gegen das Heilmittelgesetz finden sich in den Art. 86 und 87. Die meisten verwaltungsstrafrechtlichen Verfahren ${ }^{25}$ werden von Swissmedic gegen Personen geführt, die Sorgfaltspflichten im Umgang mit Heilmitteln verletzen (Art. 86 Abs. 1 lit. a HMG) oder gegen Personen, die Arzneimittel ohne Zulassung, ohne Bewilligung oder entgegen anderen Bestimmungen des Heilmittelgesetzes herstellen, in Verkehr bringen, verschreiben, einführen, ausführen oder damit im Ausland handeln (Art. 86 Abs. 1 lit. b HMG). Bei den Straftaten nach Art. 86 Abs. 1 lit. a-g HMG handelt es sich grundsätzlich um Vergehen ${ }^{26}$. Art. 86 Abs. 1 HMG verlangt in allen Tatbestandsvarianten eine konkrete Gefährdung der menschlichen Gesundheit, was aus dem Wortlaut der Bestimmung hervorgeht: «Sofern keine schwerere strafbare Handlung nach dem Strafgesetzbuch oder dem Betäubungs-

24 Kreit, S. 42.

25 Ein Überblick über die allgemeinen Entwicklungen im Heilmittelstrafrecht und die Tätigkeiten der Abteilung Strafrecht gibt der Swissmedic Geschäftsbericht 2015, S. 65 ff., abrufbar unter: www.swissmedic.ch/ueber/00134/00441/ $00445 / 00568$.

26 Art. 86 Abs. 1 HMG i.V.m. Art. 333 Abs. 1 und 2 lit. b StGB. Wird der Tatbestand jedoch gewerbsmässig begangen, handelt es sich um ein Verbrechen (Art. 86 Abs. 2 HMG i.V.m. Art. 333 Abs. 2 lit. a und Abs. 4 StGB i.V.m. Art. 10 Abs. 2 StGB; Benedikt A. Suter, BSK HMG, Vor 8. Kapitel N 23; Burri (Fn. 13), S. 93; Kreit, Anhang 4, «Aktuelle Lesart von Art. 86 HMG»). mittelgesetz vom 3. Oktober 1951 vorliegt, wird mit Freiheitsstrafe bis zu drei Jahren oder mit Geldstrafe 27 bestraft, wer die Gesundheit von Menschen gefährdet, indem er oder sie vorsätzlich: ...» ${ }^{28}$. Auch macht dies Art. $87 \mathrm{HMG}$, in welchem die heilmittelrechtlichen Übertretungen ${ }^{29}$ festgehalten sind, in Abs. 1 lit. f deutlich: Danach wird mit Busse bis zu Fr. 50'00o.--30 bestraft, «wer vorsätzlich die Tatbestände nach Artikel $86 \mathrm{Ab}-$ satz 1 erfüllt, ohne dass dadurch die Gesundheit von Menschen gefährdet wird.»

8 Die Gesundheitsgefährdung muss dabei nach der Rechtsprechung des Bundesgerichts im Einzelfall konkret nachgewiesen sein. "Eine sehr nahe konkrete Gefahr einer Gesundheitsschädigung» ${ }^{1}$ reicht nicht aus ${ }^{2}$. Widerhandlungen ge-

27 Die Strafandrohungen wurden gestützt auf Art. 333 Abs. 2-5 StGB umgerechnet; Kreit, Anhang 4 «Aktuelle Lesart von Art. $86 \mathrm{HMG}$ »; Art. 86 des Bundesgesetzes über Arzneimittel und Medizinprodukte, Änderung vom 18.3.2016, BBl 2016 1981.

28 Es handelt sich bei den Tatbeständen nach Art. 86 Abs. 1 HMG um konkrete Gefährdungsdelikte (Suter, BSK HMG, Art. 86 N 4).

29 Bei Art. 87 Abs. 2 liegt aufgrund der angedrohten Höchststrafe von Fr. 100'00o.-- ein Vergehenstatbestand vor (Art. 333 Abs. 2 lit. c i.V.m. Art. 333 Abs. 4 StGB).

3o Die Strafandrohung wurde gestützt auf Art. 333 Abs. 3 und 4 StGB umgerechnet; Kreit, Anhang 5 «Aktuelle Lesart von Art. $87 \mathrm{HMG}$; Art. 87 des Bundesgesetzes über Arzneimittel und Medizinprodukte, Änderung vom 18.3.2016, BBl 2016 1981.

31 Mit dieser Begründung befand die Strafkammer des Kantonsgerichts St. Gallen den Betroffenen u.a. des gewerbsmässigen Vergehens gegen das HMG für schuldig, begangen durch Handel mit «Viagra»; zu Unrecht, wie das BGer festhielt (vgl. BGE 135 IV 37 Lit. A.b.). Vgl. auch BGE 138 IV 57 E. 4.1.2., 4.2.2., übersetzt in Pra 2012 Nr. 67, der dieses Urteil bestätigte, sowie auch BGer-Urteil 6B_621/2015 vom 14.7.2015, E. 1.2.1.

32 BGE 135 IV 37 E. 2.4.2: Der Umstand, dass durch die unzulässige Lieferung von Viagra an eine Vielzahl von Kunden «eine sehr nahe konkrete Gefahr einer Gesundheitsschädigung» bestanden 
gen einen der in Art. 86 Abs. 1 lit. a-g HMG aufgeführten Tatbestände können aufgrund der strengen Rechtsprechung zum Nachweis der konkreten Gesundheitsgefährdung de lege lata faktisch somit lediglich als Übertretungen 33 im Sinn von Art. 87 Abs. 1 lit. f HMG bestraft werden 34 . Sowohl Art. 86 als auch Art. 87 HMG können fahrlässig begangen werden 35 .

9 Swissmedic als Verwaltungsbehörde des Bundes hat das Bundesgesetz über das Verwaltungsstrafverfahren ${ }^{36}$ anzuwen-

hat, reicht nicht aus. Vielmehr muss nachgewiesen sein, dass das Medikament bei (einem) Abnehmer einer Risikogruppe tatsächlich zu einer konkreten Gesundheitsgefahr geführt hat. Dieses Urteil wurde von Balsiger Betts, S. 56 f., zu Recht als «rechtlich noch so richtig», sachlich jedoch als «unbefriedigend» qualifiziert. Diese Rechtsprechung hat denn auch dazu geführt, dass im Rahmen der 2. Etappe der Heilmittelrevision die Strafbestimmungen des HMG dahingehend geändert wurden, dass auch bei einer rein abstrakten Gefährdung eine angemessene Bestrafung möglich wird (vgl. Art. 86 Abs. 1 des Bundesgesetzes über Arzneimittel und Medizinprodukte, Änderung vom 18.3.2016, BBl 2016 1981, der als abstraktes Gefährungsdelikt ausgestaltet wurde und Art. 86 Abs. 2, der mit der konkreten Gesundheitsgefährdung einen qualifizierten Tatbestand darstellt; Stacchetti, S. 68; Eggenberger Stöckli (Fn. 14), N 15.171); vgl. BGer-Urteil 6B_621/2015 vom 14.7.2015, E. 1.2.2.

33 Ausser es handelt sich um gewerbsmässige Begehung nach Art. 87 Abs. 2 HMG, was als Vergehen qualifiziert und mit einer Höchststrafe von 180 Tagessätzen Geldstrafe bestraft wird (Umrechnung der Strafdrohung gemäss Art. 333 Abs. 2 lit. c i.V.m. Art. 333 Abs. 4 StGB; Kreit, Anhang 5 «Aktuelle Lesart von Art. $87 \mathrm{HMG}$ ).

34 Burri (Fn. 13), S. 94. Die Widerhandlungen von Art. 87 Abs. 1 lit. f HMG sind damit subsidiär zu Art. 86 HMG und kommen nur dann zur Anwendung, wenn es an einer konkreten Gefährdung der menschlichen Gesundheit fehlt (Suter, BSK HMG, Art. $87 \mathrm{~N} 3$ ).

35 Vgl. Art. 86 Abs. 3 und Art. 87 Abs. 3 HMG; Andreas Eicker/Friedrich Frank/Jonas Achermann, Verwaltungsstrafrecht und Verwaltungsstrafverfahrensrecht, Bern 2012, S. 89.

36 VStrR; SR 313.o. den37. Für die Verfahren vor den kantonalen Gerichten gilt die Strafprozessordnung 38 , mit Ausnahme der auch für die Kantone einschlägigen Bestimmungen von Art. 6 und 7 VStrR (Widerhandlungen in Geschäftsbetrieben durch Beauftragte) 39 .

\section{Die fachtechnisch verantwortliche Person}

\section{Tätigkeitsbereich der fachtech- nisch verantwortlichen Person}

10 Sämtliche der vorgenannten SwissmedicBewilligungen werden nur erteilt, wenn die Unternehmen u.a. die «erforderlichen fachlichen Voraussetzungen» 40 erfüllen. Damit dies der Fall ist, muss dem Betrieb eine fachtechnisch verantwortliche Person ${ }^{41}$ zur Verfügung stehen ${ }^{42}$. Diese übt die unmittelbare fachliche Aufsicht über den Betrieb aus und stellt den sachgemässen Umgang mit Arzneimitteln sicher43. Darüber hinaus fallen je

37 Art. 90 Abs. 1 i.V.m. Art. 82 HMG i.V.m. Art. 1 VStrR.

38 Art. 1 Abs. 1 der Schweizerischen Strafprozessordnung (StPO; SR 312); Suter, BSK HMG, Art. $90 \mathrm{~N} 17$.

39 Art. 89 HMG; Burri (Fn. 13), S. 94.

40 Vgl. Art. 6 Abs. 1 lit. b HMG (Herstellung); Art. 19 Abs. 1 lit. a HMG (Einfuhr, Ausfuhr und Handel im Ausland); Art. 28 Abs. 2 lit. a HMG (Grosshandel); Art. 34 Abs. 2 lit. a HMG (Entnahme von Blut und Blutprodukten); Art. 27 Abs. 1 lit. b AMBV.

${ }^{41}$ Responsable technique bzw. responsabile tecnico bzw. responsible/qualified person. Es handelt sich stets um eine natürliche Person (vgl. Swissmedic, Ziff. 3 der Technischen Interpretation ISMI.TI.17 «Responsible Person: requirements» vom 1.7.2011, in der Fassung vom 23.6.2015, abrufbar unter: www.swissmedic.ch; nachfolgend: techn. Interpretation).

42 Vgl. auch Cordoba (Fn. 2), Rz 85.

43 Vgl. Art. 3 Abs. 1 lit. c i.V.m. Art. 5 AMBV (Herstellung); Art. 7 Abs. 1 lit. d i.V.m. Art. 10 AMBV (Einfuhr, Ausfuhr und Grosshandel); Art. 12 Abs. 1 lit. b i.V.m. Art. 14 AMBV (Handel im Ausland); Art. 15 Abs. 1 lit. b i.V.m. Art. 5 Abs. 1-3 AMBV (Entnahme von Blut). In diesen Bestimmungen 
nach Art der Bewilligung weitere Aufgaben in ihren Zuständigkeitsbereich, die in der Arzneimittel-Bewilligungsverordnung umschrieben werden: So wird z.B. bei der Herstellungsbewilligung verlangt, dass sie die Verantwortung für die Qualität der hergestellten Arzneimittel trägt und dafür sorgen muss, dass diese den gültigen Spezifikationen entsprechen und nach den Regeln der Guten Herstellungspraxis (Good Manufacturing Practice; GMP44) hergestellt werden 45.

11 Im Zusammenhang mit der Einfuhr-, der Ausfuhr- und der Grosshandelsbewilligung hat der fachtechnisch Verantwortliche sicherzustellen, dass die Arzneimittel nach den Regeln der Guten Vertriebspraxis (Good Distrubution Practice; GDP) vermittelt werden. Anhang 2 der Arzneimittel-Bewilligungsverordnung verweist bezüglich der auch in der Schweiz geltenden Standards insbesondere auf die einschlägigen Leitlinien der Europäischen Kommission vom 5. November 2013 für die Gute Vertriebspraxis von Humanarzneimitteln ${ }^{46}$. Im Kapitel 2 un-

regelt die AMBV auch, welche Anforderungen eine fachtechnisch verantwortliche Person in beruflicher Hinsicht erfüllen bzw. über welche Ausbildungen sie konkret verfügen muss.

44 Art. 4 Abs. 2 und Anhang 1 AMBV, der bezüglich der GMP-Regeln auf diverse internationale Richtlinien verweist. So z.B. auf die «Richtlinie 2003/94/EG der Kommission vom 8. Oktober 2003 zur Festlegung der Grundsätze und Leitlinien der Guten Herstellungspraxis für $\mathrm{Hu}-$ manarzneimittel und für zur Anwendung beim Menschen bestimmte Prüfpräparate», die in Art. 11 die «Qualitätskontrolle» regelt. Dabei hat der Hersteller ein Qualitätskontrollsystem zu betreiben, das von einer «Person mit den erforderlichen Qualifikationen» geleitet wird. In der Schweiz obliegt diese Aufgabe dem fachtechnisch Verantwortlichen.

45 Vgl. Art. 5 Abs. 2 Satz 2 AMBV.

46 Leitlinien der Europäischen Kommission vom 5 . November 2013 für die Gute Vertriebspraxis von Humanarzneimitteln (GDP Leitlinien 2013/C 343 ter Ziff. 2.2 wird der Verantwortungsbereich der fachtechnisch verantwortlichen Person umschrieben: So hat sie insbesondere für die Implementierung und Aufrechterhaltung eines Qualitätssicherungssystem zu sorgen (Ziff. i), sie hat Schulungs- und Weiterbildungsanlässe zu organisieren (Ziff. iii), Arzneimittelrückrufe zu koordinieren und durchzuführen (Ziff. iv), sicherzustellen, dass Zulieferer und Kunden über die notwendigen Bewilligungen verfügen (Ziff. vi) und sie hat zu gewährleisten, dass alle in den nationalen Rechtsvorschriften festgelegten zusätzlichen Auflagen für bestimmte Produkte eingehalten werden (Ziff. xii).

\section{Ausbildung und Vertrauenswür- digkeit}

12 Die Arzneimittel-Bewilligungsverordnung legt die Anforderungen an die Ausbildung der fachtechnisch verantwortlichen Person fest; diese variiert je nach Art der Betriebsbewilligung 47. So muss sie für die Herstellung verwendungsfertiger Arzneimittel über ein Apothekerdiplom und die notwendige Erfahrung verfügen ${ }^{48}$. In Bezug auf den Handel im

/o1; abrufbar unter: http://eur-lex.europa.eu/ legal-content/DE/TXT/PDF/?uri=CELEX:52013 XC1123(01)\&from=DE). Die GDP-Leitlinien sind Bestandteil der bilateralen Abkommen zwischen der Schweiz und der EU (vgl. dazu «Abkommen zwischen der Schweizerischen Eidgenossenschaft und der Europäischen Gemeinschaft über die gegenseitige Anerkennung von Konformitätsbewertungen»; SR 0.946.526.81).

47 Vgl. Art. 5 Abs. 3 und 4 AMBV (Herstellung); Art. 10 Abs. 2 und 3 AMBV (Einfuhr, Ausfuhr und Grosshandel); Art. 14 Abs. 2 AMBV (Handel im Ausland); Art. 15 Abs. 1 i.V.m. Art. 5 Abs. 3 AMBV (Entnahme von Blut). Vgl. auch das «Gesuch für Betriebsbewilligung - Arzneimittel» und die Ausführungen unter Ziffer 2.3 «Unterlagen zur fachtechnisch verantwortlichen Person» (abrufbar unter: www.swissmedic.ch/bewilligungen/ 00155/00242/00243/00245/00246/00247/028 27).

48 Vgl. Art. 5 Abs. 4 lit. a AMBV. 
Ausland49 schreibt die ArzneimittelBewilligungsverordnung hingegen «lediglich» vor, dass die fachtechnisch verantwortliche Person die «notwendige Ausbildung, Sachkenntnis und Erfahrung» zu belegen hat ${ }^{\circ}$. Die Technische Interpretation von Swissmedic zu den Anforderungen an die fachtechnisch verantwortliche Person sieht zudem vor, dass letztere einschlägige Erfahrung von in der Regel einem bis vier Jahren aufweisen muss, um als verantwortliche Person akzeptiert zu werden ${ }^{51}$.

Die fachtechnisch verantwortliche Person muss nicht nur hinsichtlich ihrer Ausbildung den Anforderungen der Arzneimittel-Bewilligungsverordnung genügen, sondern sie hat darüber hinaus auch «vertrauenswürdig» zu sein. Die Vertrauenswürdigkeit wird verneint, wenn die Person wegen eines Verstosses gegen das Heilmittelgesetz, gegen das Betäubungsmittelgesetz ${ }^{22}$ oder gegen andere mit diesen Erlassen im Zusammenhang stehende Vorschriften rechtskräftig verurteilt wurde53. Swissmedic darf zur Überprüfung der Vertrauenswürdigkeit einen Strafregisterauszug verlangen54.

$49 \overline{\text { Handel im Ausland liegt vor, wenn von der }}$ Schweiz aus mit Arzneimitteln gehandelt wird, ohne dass diese das Gebiet der Schweiz berühren (vgl. Art. 18 Abs. 1 lit. c HMG).

50 Vgl. Art. 14 Abs. 2 AMBV.

51 Vgl. Swissmedic, Ziff. 5.2 der techn. Interpretation.

52 Bundesgesetz über die Betäubungsmittel und die psychotropen Stoffe (Betäubungsmittelgesetz; BetmG; SR 812.121).

$53 \mathrm{Vgl}$. Ziff. 5.3 der techn. Interpretation.

54 Vgl. Art. 5 Abs. 3 und 4 AMBV (Herstellung); Art. 10 Abs. 2 und 3 AMBV (Einfuhr, Ausfuhr und Grosshandel); Art. 14 Abs. 2 AMBV (Handel im Ausland); Art. 15 Abs. 1 i.V.m. Art. 5 Abs. 3 AMBV (Entnahme von Blut). Vgl. auch das «Gesuch für Betriebsbewilligung - Arzneimittel» und die Ausführungen unter Ziffer 2.3 «Unterlagen zur fachtechnisch verantwortlichen Person» (abrufbar unter: www.swissmedic.ch/bewilligungen/

\section{Weisungsbefugnis und Unab- hängigkeit}

14 Unabhängig von der Art der Bewilligung schreibt die Arzneimittel-Bewilligungsverordnung vor, dass die fachtechnisch verantwortliche Person in ihrem Bereich «weisungsbefugt» 55 sein muss. Dies bedeutet z.B. mit Blick auf die Herstellung von Arzneimitteln, dass sie unabhängig von der Geschäftsleitung des Unternehmens über die Freigabe einer Charge ${ }^{5}$ muss entscheiden können57. Die Bewilligungsinhaberin ist verpflichtet, in sog. Standardarbeitsanweisungen ( $«$ Standard Operation Procedures»; SOP) die Befugnisse, betriebsinternen Aufgaben und Verantwortlichkeiten ihrer fachtechnisch verantwortlichen Person umfassend und schriftlich zu regeln und diese Swissmedic im Rahmen einer verwaltungsrechtlichen Inspektion $5^{8}$ auf Verlangen vorzuweisen 59.

00155/00242/00243/00245/00246/00247/ 02827).

55 Vgl. Art. 5 Abs. 1 AMBV (Herstellung); Art. 10 Abs. 1 AMBV (Einfuhr, Ausfuhr und Grosshandel); Art. 14 Abs. 1 AMBV (Handel im Ausland); Art. 15 Abs. 1 lit. b i.V.m. Art. 5 Abs. 1 AMBV (Entnahme von Blut).

56 Nach Art. 2 lit. c AMBV ist eine «Charge: eine in einem Arbeitsgang oder in einer Reihe von Arbeitsgängen gefertigte, homogene und definierte Menge.»

57 Vgl. Art. 5 Abs. 1 und 2 AMBV; vgl. dazu auch Cordoba (Fn. 2), Rz 87, 89.

58 Mit der Zulassung (dazu Art. 9 ff. HMG) oder der Erteilung einer Betriebsbewilligung ist die Tätigkeit von Swissmedic nicht abgeschlossen. Das Institut und die Kantone überwachen im Rahmen ihrer Zuständigkeiten auch danach weiterhin die Rechtmässigkeit der Herstellung, des Vertriebs, der Abgabe und der Anpreisung von Heilmitteln. Dabei überprüfen sie mit periodischen Inspektionen, ob die Voraussetzungen für die Bewilligungen noch erfüllt sind (vgl. Art. 58 Abs. 1 und Art. $60 \mathrm{HMG})$.

59 Vgl. Swissmedic, Ziff. 5.4 der techn. Interpretation. 
Während der Geschäftsleiter6o des Unternehmens die administrativen, personellen und finanziellen Belange des Unternehmens $\mathrm{zu}$ besorgen hat, liegt die fachliche Verantwortung für die Herstellung oder den Handel mit Arzneimitteln bei der fachtechnisch verantwortlichen Person. Zwar lässt es Swissmedic mitunter $\mathrm{zu}$, dass die fachtechnisch verantwortliche Person Einsitz in der Geschäftsleitung hat ${ }^{61}$; dass die Funktionen des Geschäftsleiters und der fachtechnisch verantwortlichen Person eines Pharmaunternehmens bei der gleichen Person zusammenfallen, ist im Regelfall jedoch ausgeschlossen: Das Interesse an einem finanziell gesunden bzw. florierenden Unternehmen kann mit den Sicherheitsvorschriften der Heilmittelgesetzgebung konkurrieren und damit zu einer Interessenkollision führen ${ }^{62}$, weshalb eine Personalunion von Geschäftsführer bzw. Produktionsleiter und fachtechnisch verantwortlicher Person ausscheidet 63 .

$60 \overline{\text { Der Begriff des Geschäftsleiters ist im Heilmittel- }}$ recht weiter auszulegen als im Obligationenrecht. Er umfasst nicht nur die gemäss Organigramm als Geschäftsleiter bezeichnete Person, sondern vielmehr sämtliche Personen, die die "geschäftlich relevanten wirtschaftlichen Entscheidungen für die Bewilligungsinhaberin» treffen (VPB 70.22, Auszug aus dem Entscheid HM 04.067 der Eidg. Rekurskommission für Heilmittel vom 11.4.2005).

61 Vgl. Swissmedic, Ziff. 5.4 der techn. Interpretation.

$62 \mathrm{Zu}$ denken ist zum Beispiel daran, dass eine Charge bei der Herstellung verunreinigt wurde. Aus sicherheitspolizeilicher Sicht darf die fachtechnisch verantwortliche Person die Charge nicht freigeben (vgl. Art. 5 Abs. 2 AMBV). Demgegenüber mag der Geschäftsleiter aus finanziellen Gründen kein Interesse daran haben, die Charge nicht weiterverwenden bzw. verkaufen zu können.

63 Swissmedic, Ziff. 5.4 der techn. Interpretation.

\section{Präsenzzeiten und Stellvertretung}

16 Die Praxis von Swissmedic zur Arzneimittel-Bewilligungsverordnung lässt es $\mathrm{zu}$, dass die fachtechnisch verantwortliche Person in einem Teilzeitverhältnis arbeitet $^{64}$ oder in unterschiedlichen Unternehmen in dieser Funktion tätig ist ${ }^{65}$. Es muss aber sichergestellt sein, dass sie im Bedarfs- oder Notfall das Unternehmen innert kurzer Frist erreicht ${ }^{66}$.

17 Die Stellvertretung der fachtechnisch verantwortlichen Person muss durch Fachleute ausreichender Qualifikation sichergestellt sein ${ }^{67}$. Die fachlichen Anforderungen an die Stellvertretung müssen - um den faktischen Verhältnissen kleinerer Unternehmen Rechnung zu tragen - nicht notwendigerweise denen der fachtechnisch verantwortlichen Person entsprechen ${ }^{68}$. Die Verantwortung dafür, dass nur qualitativ hochstehende, sichere und wirksame Arzneimittel in den Verkehr gebracht werden, liegt jedoch stets bei der fachtechnisch verantwortlichen Person.

\section{Zusammenfassung}

18 Die Funktion der fachtechnisch verantwortlichen Person ist anspruchsvoll und mit weitreichenden Befugnissen bzw. Verpflichtungen verbunden. Entspre-

64 Vgl. Art. 5 Abs. 6 AMBV (Herstellung); Art. 10 Abs. 4 AMBV (Einfuhr, Ausfuhr und Grosshandel); Art. 14 Abs. 3 AMBV (Handel im Ausland). Die minimale Präsenzzeit darf $10 \%$ eines Vollzeitpensums grundsätzlich nicht unterschreiten (Swissmedic, Ziff. 5.6 der techn. Interpretation).

65 Swissmedic, Ziff. 5.6 der techn. Interpretation.

66 Swissmedic, Ziff. 5.5 der techn. Interpretation.

67 Vgl. Art. 5 Abs. 7 AMBV (Herstellung); Art. 10 Abs. 5 AMBV (Einfuhr, Ausfuhr und Grosshandel); Art. 14 Abs. 4 AMBV (Handel im Ausland).

68 Keine Kompromisse werden bei der Vertrauenswürdigkeit der stellvertretenden Fachleute gemacht (Swissmedic, Ziff. 5.7 der techn. Interpretation). 
chende Berufserfahrung und einschlägige Vorkenntnisse sind daher unabdingbar. Dies gerade auch mit Blick auf die erhöhte strafrechtliche Verantwortlichkeit, die mit der Funktion als fachtechnisch verantwortliche Person einhergeht, wie die nachfolgenden Ausführungen zeigen.

\section{Strafrechtliche Verantwortlichkeit der fachtechnisch verantwortlichen Person}

Zur Illustration diene folgender, einfacher Sachverhalt: Die X AG verfügt seit Jahrzehnten über eine SwissmedicBewilligung zur Ausfuhr von Arzneimitteln 69 . Da sie die Arzneimittel zu humanitären Zwecken in Entwicklungsländer exportiert, erhält sie von Pharmahändlern vorteilhafte Konditionen und kann die Arzneimittel zu vergünstigten Preisen ${ }^{70}$ erwerben. Darüber hinaus betreibt die X AG aber seit Kurzem zusätzlich Grosshandel in der Schweiz: Sie verkauft die für die humanitäre Ausfuhr vorgesehenen Arzneimittel an verschiedene Pharmagrossisten mit Sitz in der Schweiz weiter. Einen Teil des Rabatts, den die X AG für die vermeintlichen humanitären Ausfuhren von den Händlern erhält, gibt sie an ihre Abnehmer, Schweizer Pharmagrossisten, weiter. Letztere können daher Arzneimittel bei der X AG preiswerter einkaufen, als wenn sie diese über ihre bisherigen Lieferanten beziehen

69 Vgl. Art. 18 Abs. 1 lit. b HMG.

70 Bei der Preisbildung von Arzneimitteln gilt es in aller Kürze - Folgendes zu unterscheiden: Bei kassenpflichtigen Arzneimitteln wird in der Regel auf den in der Spezialitätenliste des Bundesamts für Sozialversicherungen aufgeführten «exfactory-Preis» abgestellt (vgl. www.spezialitätenliste.ch). Bei nicht kassenpflichtigen Arzneimitteln und damit solchen, die auf der Spezialitätenliste nicht aufgeführt sind, erfolgt die Preisbildung nach den Marktregeln. würden. Die X AG selbst erzielt mit der Differenz entsprechend einen Gewinn. Allerdings verfügt sie für diese Geschäftstätigkeit nicht über die dafür zwingend notwendige Grosshandelsbewilligung ${ }^{71}$ von Swissmedic.

20 Damit verstösst sie gegen Art. 86 Abs. 1 lit. b HMG, weil sie Arzneimittel ohne Bewilligung in Verkehr bringt ${ }^{72}$. Es fehlt jedoch an einer konkreten Gesundheitsgefährdung: Die Arzneimittel, die die X AG innerhalb der Schweiz vertreibt, sind qualitativ einwandfrei und genügen den heilmittelrechtlichen Vorschriften. Eine konkrete Gesundheitsgefährdung im Sinn des Gesetzes ist folglich zu verneinen. Die X AG bzw. die verantwortlichen Personen machen sich damit nach Art. 86 Abs. 1 lit. b i.V.m. Art. 87 Abs. 1 lit. f HMG strafbar, weil sie Arzneimittel ohne Bewilligung in Verkehr bringt, ohne dabei die Gesundheit von Menschen konkret zu gefährden (abstrakte Gesundheitsgefährdung) 73 .

21 Im Zusammenhang mit der Strafverfolgung der fachtechnisch verantwortlichen Person werden in der Praxis häufig folgende Szenarien angetroffen:

71 Vgl. Art. 28 f. HMG.

72 «Inverkehrbringen» ist das Vertreiben und Abgeben von Heilmitteln, wobei «Vertreiben» die entgeltliche oder unentgeltliche Übertragung oder Überlassung eines Heilmittels mit Ausnahme des Abgebens darstellt (vgl. Art. 4 Abs. 1 lit. $d$ und e HMG).

73 Auch die fahrlässige Tatbegehung ist strafbar (Art. 86 Abs. 1 lit. b i.V.m. Art. 86 Abs. 3 HMG bzw. Art. 87 Abs. 1 lit. f i.V.m. Art. 87 Abs. 3 HMG i.V.m. Art. 28 HMG). Das Verhalten der X AG bzw. deren Organe wäre auch unter dem Tatbestand des Betrugs (Art. 146 StGB) zum Nachteil ihrer Lieferantinnen zu prüfen. In vorliegendem Aufsatz werden aber ausschliesslich die heilmittelrechtlichen Aspekte untersucht. 


\section{Handlungsdelikt}

22 Dem Verantwortlichen F obliegt u.a. die Prüfung, ob die von der X AG vorgenommenen Geschäftstätigkeiten mit den von Swissmedic erteilten Bewilligungen übereinstimmen74. Angenommen, F überprüft den Bewilligungsumfang seiner Arbeitgeberin und kommt zu Recht zum Schluss, dass der Grosshandel mit Arzneimitteln in der Schweiz von der Ausfuhr-Bewilligung von Swissmedic nicht gedeckt ist; trotzdem genehmigt er den Handel der X AG mit den Pharmagrossisten in der Schweiz. Aus strafrechtlicher Sicht handelt es sich um die einfachste der denkbaren Szenarien: F hat den Tatbestand der illegalen Grosshandelstätigkeit nach Art. 86 Abs. 1 lit. b i.V.m. Art. 87 Abs. 1 lit. f HMG in eigener Person vorsätzlich erfüllt und hat sich strafbar gemacht 75 .

23 In der Praxis wird von der fachtechnisch verantwortlichen Person in einer solchen Konstellation häufig geltend gemacht, sie habe die Grosshandelstätigkeit der X AG nur deshalb genehmigt, weil sie davon ausgegangen sei, der Grosshandel in der Schweiz sei durch die Ausfuhrbewilligung mitabgedeckt. Mit anderen Worten habe sie nicht vorsätzlich, sondern «nur» fahrlässig gehandelt 76 .

74 Vgl. Art. 10 Abs. 1 Satz 1 AMBV. Auf der Webseite von Swissmedic findet sich eine öffentlich zugängliche Liste, auf welcher sämtliche Bewilligungsinhaber aufgeführt sind. Es lässt sich damit feststellen, welches Unternehmen über welche Swissmedic-Betriebsbewilligung(en) verfügt: www.swissmedic.ch/bewilligungen/o0155/ 00241/00253.

75 Vgl. auch Cordoba (Fn. 2), Rz 90.

76 Es handelt sich hier um die sog. "Rechtsfahrlässigkeit» und nicht um «Tatfahrlässigkeit». Von «Rechtsfahrlässigkeit» wird gesprochen, wenn die Strafbarkeit im Nebenstrafrecht nicht auf die pflichtwidrig unvorsichtige Begehung der Tat (vgl. dazu Art. 12 Abs. 3 StGB), sondern «auf die
24 Die fachtechnisch verantwortliche Person ist naturwissenschaftlich ausgebildet und verfügt über mehrere Jahre Berufserfahrung in der Pharmabranche. Es stellt eine ihrer Hauptaufgaben dar, zu gewährleisten, dass sich die Tätigkeiten des Unternehmens innerhalb des von den Aufsichtsbehörden festgelegten Rahmens abspielen. Mit dieser Überwachungsaufgabe sind keine besonderen Schwierigkeiten verbunden: In der Verfügung von Swissmedic werden die bewilligen Tätigkeiten - Herstellung, Einfuhr, Grosshandel in der Schweiz, Ausfuhr, Handel mit Arzneimitteln im Ausland77 - eindeutig festgelegt. Zudem genügt ein Blick auf die von Swissmedic im Internet publizierte Liste der Bewilligungsinhaber, um sich über den Bewilligungsumfang des Unternehmens Klarheit zu verschaffen. Liegen keine aussergewöhnlichen Umstände vor, wird die fachtechnisch verantwortliche Person mit dem Einwand, sie habe angenommen, auch eine andere als in der Verfügung von Swissmedic bezeichnete Tätigkeit sei von der Bewilligung erfasst, nicht durchdringen.

vorwerfbare Verbotsunkenntnis abzielt» (Marc Thommen, Art. 333 Abs. 7 StGB - Grenzenlose Fahrlässigkeit im Nebenstrafrecht?, in: recht 2013, S. 276 ff., S. 283; dazu auch Guido Jenny, Tatbestands- und Verbotsirrtum im Nebenstrafrecht, in: ZStrR 2/1990, S. 241 ff.). Nach heutiger Auffassung sind die Fälle pflichtwidriger Verbotsunkenntnis durch Art. 21 StGB abge-deckt (Thommen, S. 286; zur historischen Bedeutung und der heutigen Berechtigung von Art. 333 Abs. 7 StGB bzw. der Fahrlässigkeit im Nebenstrafrecht vgl. ausführlich Thommen, passim).

77 Jeweils mit weiterer Untergliederung bei der Herstellung, der Einfuhr und dem Grosshandel, vgl. dazu: www.swissmedic.ch/bewilligungen/ 00155/00241/00253. 


\section{Unterlassungsdelikt (Art. 11 StGB)}

25 Obwohl F im Rahmen seiner Überwachungspflichten festgestellt hat, dass die $\mathrm{X}$ AG Grosshandel in der Schweiz betreibt, ohne über die hierfür notwendige Grosshandelsbewilligung zu verfügen, interveniert er nicht. Weder gebietet er der Grosshandelstätigkeit Einhalt noch genehmigt er diese aktiv, er lässt die Geschäftsleitung der X AG schlicht gewähren.

In der ersten Konstellation hat $\mathrm{F}$ den objektiven Tatbestand durch eine aktive Handlung erfüllt. An eine aktive Handlung kann hier jedoch gerade nicht angeknüpft werden, weshalb zu prüfen ist, ob $\mathrm{F}$ den Tatbestand des illegalen Grosshandels mit Arzneimitteln in der Schweiz durch Unterlassen erfüllt hat. Beim - wie hier vorliegenden - unechten Unterlassungsdelikt ist zu prüfen, ob das Unterlassen einer Handlung gleich behandelt werden soll wie die aktive Begehung eines Delikts 78 . Die Gleichbehandlung ist dann möglich, wenn dem Täter eine spezifische Handlungspflicht obliegt, aufgrund welcher er für den Schutz eines bestimmten Rechtsguts zu sorgen hat 79 . Diese Handlungspflicht ergibt sich aus einer Garantenstellung, die sich aus einer besonderen Rechtspflicht ableitet ${ }^{80}$.

Unstrittig ist, dass die hier vorliegende Übertretung grundsätzlich auch durch «pflichtwidriges Untätigwerden» 81 begangen werden kann. Nach dem Gesetz bleibt pflichtwidrig untätig, «wer die Gefährdung oder Verletzung eines straf-

78 Diego R. Gfeller, Die Privatbestechung, Art. 4a UWG, Konzeption und Kontext, Diss., Basel 2010, S. 263.

79 Gfeller (Fn. 78), S. 263 f.

80 Gfeller (Fn. 78), S. 264.

81 Vgl. Art. 11 Abs. 1 i.V.m. Art. 104 StGB. rechtlich geschützten Rechtsgutes nicht verhindert, obwohl er aufgrund seiner Rechtstellung dazu verpflichtet ist» 82 . Mit anderen Worten muss F eine Garantenstellung zukommen, wobei vorliegend eine solche aus «Gesetz» nach Art. 11 Abs. 2 lit. a StGB in Betracht kommt. Allerdings begründet nach der Rechtsprechung des Bundesgerichts nicht jede gesetzliche Pflicht eine entsprechende Garantenstellung ${ }^{83}$, vielmehr bedarf es einer entsprechenden «Rechtsstellung» des Betroffenen ${ }^{8} 4$ bzw. eine besondere Rechtspflicht. Letztere ergibt sich für die fachtechnisch verantwortliche Person aus Art. 10 Abs. 1 AMBV. Diese Bestimmung sieht u.a. bezüglich des Grosshandels in der Schweiz vor, dass die fachtechnisch verantwortliche Person die unmittelbare fachliche Aufsicht über den Betrieb auszuüben und insbesondere den sachgemässen Umgang mit Arzneimitteln sicherzustellen hat ${ }^{85}$. Dafür ist das Unternehmen bzw. der vom Unternehmen im Gesuch bezeichnete fachtechnisch Verantwortliche mit Erteilung der Swissmedic-Bewilligung verantwortlich. Mit anderen Worten wird dem Verantwortlichen in einem bestimmten Bereich von Swissmedic die Pflicht überbunden, sicherzustellen, dass die von «seinem» Unternehmen hergestellten oder gehandelten Arzneimittel den gesetzlichen Vorga-

82 Art. 11 Abs. 2 StGB.

83 Kurt Seelmann, in: BSK zum Strafrecht I, Art. 1110 StGB, Jugendstrafgesetz, hrsg. von Marcel Alexander Niggli/Hans Wiprächtiger, 3. Aufl., Basel 2013, Art. 11 N 34; BGE 118 IV 309 E. 1d; 120 IV 98 E. 2c; BGer-Urteil 6B_1026/2008 vom 1.5.2009, E. 2.3.4.

84 Seelmann, BSK StGB I, Art. $11 \mathrm{~N} 34$.

85 Gleiches gilt auch für die Herstellung (Art. 5 Abs. 1 AMBV), für den Handel im Ausland (Art. 14 Abs. 1 AMBV) und für die besonderen Bestimmungen im Zusammenhang mit der Bewilligung zur Entnahme von Blut (Art. 15 Abs. 1 lit. b i.V.m. Art. 5 Abs. 1 AMBV). 
ben entsprechen. Anhang 2 der Arzneimittel-Bewilligungsverordnung verweist zudem auf die GDP-Leitlinien der EU86. In Art. 2.2 dieser Leitlinien sind die Pflichten der fachtechnisch verantwortlichen Person detailliert aufgeführt; dazu gehört es auch, sicherzustellen, dass sie sich auf die «Durchführung genehmigter Tätigkeiten ${ }^{87}$ konzentriert. Sie muss also gewährleisten, dass sich die Tätigkeiten ihrer Arbeitgeberin im Rahmen des heilmittelrechtlich Bewilligten abspielen. Aus diesen Bestimmungen und Pflichten lässt sich eine besondere Rechtsstellung der fachtechnisch verantwortlichen Person und damit eine Garantenpflicht ableiten ${ }^{88}$. Denn zu überwachen, ob die heilmittelrechtlichen Vorschriften bei den einzelnen Handlungen ihrer Arbeitgeberin eingehalten werden, stellt nach dem Gesagten ausdrücklich eine der zentralen Aufgaben der fachtechnisch verantwortlichen Person dar. Entsprechend kommt letzterer eine Garantenstellung aus Gesetz zu, weshalb sie auch bei unterlassener aber hier gebotener wie zumutbarer Intervention den Tatbestand des illegalen Grosshandels mit Arzneimitteln erfüllt.

\section{Geschäftsherrenhaftung (Art. 6 Abs. 2 und 3 VStrR)}

Angenommen, $\mathrm{F}$ gibt im Rahmen der Einvernahme im Verwaltungsstrafverfahren von Swissmedic zu Protokoll, nicht er habe die Grosshandelstätigkeit der X AG genehmigt, sondern sein Mitarbeiter $\mathrm{M}$.

86 Vgl. dazu vorstehende Fn 49.

87 Art. 2.2 (ii) der EU-Leitlinien zur GDP.

88 Vgl. auch Suter, BSK HMG, Art. 86 N 41; Burri (Fn. 13), S. 133 Fn 158; Cordoba (Fn. 2), Rz 91, der zudem in Rz 92 ausführt, dass sich eine Garantenstellung der fachtechnisch verantwortlichen Person auch aus Ingerenz ergeben könne.
29 Neben der Bestrafung des Haupttäters M ist zu prüfen, ob F ebenfalls strafrechtlich zur Verantwortung gezogen werden muss, weil er in seiner Funktion als fachtechnisch verantwortliche Person die Tätigkeiten seiner hierarchisch Untergeordneten nicht genügend überwacht hat ${ }^{89}$. Zur Diskussion steht damit die Geschäftsherrenhaftung nach Art. 6 Abs. 2 VStrR, wonach der Geschäftsherr, Arbeitgeber, Auftraggeber oder Vertretene, der es vorsätzlich oder fahrlässig in Verletzung einer Rechtspflicht unterlässt, eine Widerhandlung des Untergebenen, Beauftragten oder Vertreters abzuwenden oder in ihren Wirkungen aufzuheben, den Strafbestimmungen untersteht, die für den entsprechend handelnden Täter gelten. Für Unternehmen, wobei es sich im Anwendungsbereich des Heilmittelgesetzes in aller Regel um juristische Personen handelt, bestimmt Art. 6 Abs. 3 VStrR, dass als Geschäftsherr u.a. auch Organe und «tatsächlich leitende Personen» gelten. Die vorgesetzten Personen stehen damit in der Pflicht, den rechtmässigen Zustand aufrecht zu erhalten bzw. wenn eine Störung bereits eingetreten ist, diese zu beseitigen ${ }^{\circ}$. Die Geschäftsherrenhaftung bezweckt, Führungspersonen eines Unternehmens zu bestrafen, die wissen oder annehmen müssen, dass ihre Untergebenen Straftaten begehen und diese Straftaten nicht unterbinden ${ }^{11}$. Sie wurde zum einen deshalb ins Gesetz aufgenommen, weil das Nichtverhindern von Straftaten durch Vorgesetzte als strafwürdig erachtet wurde $^{92}$ und, zum anderen, weil sich das

89 Burri (Fn. 13), S. 132.

90 Burri (Fn. 13), S. $132 \mathrm{f}$.

91 Eicker/Frank/Achermann (Fn. 35), S. 51.

92 Vgl. in diesem Zusammenhang die bekannten BGE «Bührle» (96 IV 155) und «Von Roll» (122 IV 103), die letztlich zum Erlass von Art. 11 StGB 
Verhalten des Geschäftsherrn nicht klar den vorhandenen Kategorien von Täterschaft und Teilnahme zuordnen lässt93.

Um gestützt auf die Geschäftsherrenhaftung bestraft werden zu können, muss F im konkreten Fall eine Sonderstellung als Geschäftsherr, Arbeitgeber, Auftraggeber oder Vertretener zukommen94: F ist weder Arbeitgeber noch Auftraggeber oder Vertretener und steht mit $M$ in keinem (arbeits-)vertraglichen Verhältnis. Was den Begriff des «Geschäftsherrn» betrifft, verstehen Eicker/Frank/Achermann Organe und natürliche Personen darunter, «welchen die Gesamtgeschäfts-

(in Kraft seit 1.1.2007) geführt haben. Dazu und weiterführend: Cordoba (Fn. 2), Rz 69 f.; Eicker/Frank/Achermann (Fn. 35), S. 55 ff.; Fabio Burgener, La responsabilité pénale du chef d'entreprise, in: ZStrR 133/2015, S. 368-398, S. 373 ff.; Andrew M. Garbarski, L'entreprise dans le viseur du droit pénal administratif: éléments de droit matériel et de procédure, in: ZStrR 130/2012, S. 409-446, S. 414 f. (nachfolgend: Garbarski, 2012); Andrew M. Garbarski, La responsabilité civile et pénale des organes dirigeants de sociétés anonymes, Diss. Lausanne 2006, Zürich 2006, S. 328 f. (nachfolgend: Garbarski, 2006); Andrew M. Garbarski/Alain Macaluso, La responsabilité de l'entreprise et de ses organes dirigeants à l'épreuve de droit pénal administratif, in: AJP 7/2008, S. 833-846, S. 838.

93 Eicker/Frank/Achermann (Fn. 35), S. 51, mit weiteren Ausführungen dazu, weshalb beim Geschäftsherrn weder Gehilfenschaft (fehlender untergeordneter Tatbeitrag), noch Anstiftung (fehlendes Hervorrufen des Tatentschlusses beim Haupttäter), mittelbare Täterschaft (kein «Vorsatzdefekt» beim unmittelbaren Täter und daher keine Tatherrschaft) oder Mittäterschaft (keine Tatherrschaft) vorliegt; Friedrich Frank, Zur Verteidigung im Verwaltungsstrafverfahren, in: AJP 9/2012, S. 1266-1280, S. 1276; Gfeller (Fn. 78), S. 263; vgl. auch Kurt Hauri, Verwaltungsstrafrecht, Bundesgesetz vom 22. März 1974 über das Verwaltungsstrafrecht, Motive - Doktrin - Rechtsprechung, Bern 1998, Art. 6 N 8a, 12a, b.

94 Es handelt sich bei der Geschäftsherrenhaftung um ein echtes Sonderdelikt (Eicker/Frank/Achermann (Fn. 35), S. 52; Gfeller (Fn. 78), S. 264). führung in operativer Hinsicht zukommt, wie z.B. den Mitgliedern eines Verwaltungsrats einer $A G »$; diese auf den ersten Blick etwas eng anmutende Definition wird in der Folge zu Recht dahingehend ergänzt, dass jede Person, die «gegenüber dem unmittelbaren Täter eine Überwachungspflicht hat», als Geschäftsherr im Sinn des Verwaltungsstrafrechtsgesetzes $\mathrm{zu}$ verstehen ist 95 . Nach Burgener ist Geschäftsherr derjenige, dem Entscheidbefugnisse und Überwachungspflichten zukommen ${ }^{96}$. Eine ähnliche Definition findet sich auch bei Gfeller, wonach Geschäftsherr derjenige ist, dem gegenüber dem Täter eine Überwachungspflicht zukommt ${ }^{97}$. Garbarski definiert den Geschäftsherrn als Person, die ihren Untergebenen Anweisungen erteilen kann ${ }^{98}$. Hier ist F gegenüber $M$ in einem bestimmten Bereich direkt und unmittelbar weisungsbefugt sowie hierarchisch übergeordnet 99 . Zudem obliegen ihm als fachtechnisch verantwortliche Person entsprechende Überwachungspflichten, die er völlig unabhängig von der Unternehmensstruktur wahrzunehmen hat. F kommt damit die Stellung eines Geschäftsherrn im Sinn von Art. 6 Abs. 2 (i.V.m. Art. 6 Abs. 3) VStrR zu.

95 Eicker/Frank/Achermann (Fn. 35), S. 52 f.

96 Burgener, S. 376.

97 Gfeller (Fn. 78), S. 267, mit weiteren Hinweisen; vgl. zum Ganzen auch Cordoba (Fn. 2), Rz 80 ff.

98 Garbarski, 2006, S. 331, mit weiteren Hinweisen.

99 Vgl. Garbarski/Macaluso, S. 836 f.; Cordoba (Fn. 2), Rz 102; Garbarski, 2012, S. 415. Hingegen fällt eine Bestrafung gestützt auf Art. 6 Abs. 2 VStrR ausser Betracht, wenn der Geschäftsherrn dem unmittelbaren Täter hierarchisch gleichgestellt oder gar untergeordnet ist (BGE 113 IV 68 E. 6e; vgl. auch Burri (Fn. 13), S. 132 Fn 151; a.M.: Gfeller (Fn. 78), S. 268, wonach für eine Bestrafung des Geschäftsherrn weder Subordinationsverhältnis noch Weisungsbefugnis vorausgesetzt werden). 
31 Strafbar macht sich ein Geschäftsherr wegen der Nichtvornahme einer gebotenen und zumutbaren Handlung, womit deutlich wird, dass es sich bei der Geschäftsherrenhaftung um ein unechtes Unterlassungsdelikt handelt ${ }^{100}$. Entsprechend bildet wie bereits unter IV.2. auch hier die Verletzung einer Garantenpflicht ${ }^{101}$ eine unabdingbare Voraussetzung für die Bestrafung des Geschäftsherrn ${ }^{102}$. Diesbezüglich wurde bereits aufgrund vorstehender Ausführungen deutlich, dass der fachtechnisch verantwortlichen Person in ihrem Kompetenzbereich eine Garantenstellung zukommt, die sich hier für $\mathrm{F}$ aus Art. 10 Abs. 1 AMBV ergibt ${ }^{103}$ und nach welcher es ihre Aufgabe ist, in ihrem Zuständigkeitsbereich die öffentliche Gesundheit zu schützen. Einschränkend gilt festzuhalten, dass sich die Garantenpflicht des Geschäftsherrn nur auf diejenigen Risiken beschränken kann, die betriebsspezifisch sind; mit anderen Worten haftet der Geschäftsherr nur für die Verletzung derjenigen Rechtsgüter, die er aufgrund seiner Funktion gerade schützen soll104, wie vorliegend z.B. die öffentliche Gesundheit. F würde daher für den Diebstahl des $M$ an einem Arbeitskollegen

$100 \overline{\text { Markus Oertle, Die Geschäftsherrenhaftung im }}$ Strafrecht, Unter besonderer Berücksichtigung von Art. 6 Abs. 2 des Bundesgesetzes über das Verwaltungsstrafrecht (VStrR), Diss., Zürich 1996, S. 179; Gfeller (Fn. 78), S. 263.

101 Die Geschäftsherrenhaftung wird auch als «Garantenhaftung» bezeichnet (Eicker/Frank/ Achermann, S. 51).

102 Eicker/Frank/Achermann (Fn. 35), S. 53; Renate Schwob, Verwaltungsstrafrecht des Bundes I-V, in: SJK Karten 1985 [Schweizerische Juristische Kartothek], Karten Nrn. 1286-1290, Nr. 1287, S. 5; Cordoba (Fn. 2), Rz 82; Burgener, S. 377.

103 Vgl. dazu vorne V.2.b. Zum Lehrstreit, woraus die Garantenpflicht abzuleiten ist: Eicker/Frank/ Achermann (Fn. 35), S. 53 f. m.w.H.; Burri (Fn. 13), S. 132 ff. m.w.H.

104 Eicker/Frank/Achermann (Fn. 35), S. 54. nicht strafrechtlich zur Verantwortung gezogen werden können. Denn als fachtechnisch verantwortliche Person obliegt es ihm, in seinem Tätigkeitsbereich für sichere Heilmittel zu sorgen. Dies beinhaltet selbstredend nicht, für Eigentumsdelikte seines Mitarbeiters M (strafrechtlich) gerade zu stehen ${ }^{105}$.

32 Die Bestrafung gestützt auf Art. 6 Abs. 2 VStrR setzt weiter voraus, dass der Geschäftsherr es unterlassen hat, die Widerhandlung eines Untergebenen abzuwenden oder in ihren Wirkungen aufzuheben $^{106}$. Bei der Frage der Vermeidbarkeit der Widerhandlungen wird der Massstab streng angesetzt, wie das Urteil 6B_444/2010 vom 16.9.2010 des Bundesgerichts illustriert: Pharmazeutische Unternehmen, die (ausschliesslich) über eine Bewilligung zum Grosshandel mit Arzneimitteln verfügen, dürfen Arzneimittel nur an Unternehmen liefern, die ihrerseits entweder über eine Grosshandelsbewilligung verfügen ${ }^{107}$ oder die befugt sind, Arzneimittel an den Endverbraucher abzugeben ${ }^{108}$. Letzteres ist z.B. bei Apotheken sowie bei Ärzten der Fall, die über eine kantonale Bewilligung zur Selbstdispensation verfügen. Es ist Aufgabe der fachtechnisch verantwortlichen Person sicherzustellen, dass Arzneimittel

105 Vgl. Cordoba (Fn. 2), Rz 102; Burgener, S. 379; Gfeller (Fn. 78), S. 268; BGE 113 IV 68 E. 6d und 7 («sektorale Garantenstellung»).

106 Damit wird deutlich, dass die fachtechnisch verantwortliche Person auch dann intervenieren muss, wenn sie von der Straftat erst im Nachhinein erfährt (vgl. Oertle (Fn. 100), S. 196; Eicker/Frank/Achermann (Fn. 35), S. 54; Hauri (Fn. 93), Art. 6 N 1ob; Schwob, Nr. 1287, S. 5 f.; Gfeller (Fn. 78), S. 269).

107 Dies lässt sich mit einem Blick auf die Swissmedic-Liste der Bewilligungsinhaber feststellen. Unternehmen, die über eine Grosshandelsbewilligung verfügen, sind in der Kolonne $« \mathrm{C}$ » verzeichnet.

108 Vgl. dazu auch Lachat-Boillat/Stacchetti, S. 223. 
nur an bezugsberechtigte juristische oder natürliche Personen abgegeben werden. Im konkreten Fall gelangte ein Arzt mit einer psychiatrischen Allgemeinpraxis an das Unternehmen und bestellte eine grosse Menge an Arzneimitteln aus unterschiedlichsten Gebieten. Der Arzt verfügte jedoch nicht über eine Bewilligung zur Selbstdispensation, sondern ihm wurde von den kantonalen Behörden lediglich erlaubt, Arzneimittel für Notfälle und für die «Direktversorgung» abzugeben. Die vom Arzt beim Pharmaunternehmen bestellte Anzahl an Arzneimitteln überschritt zum einen die durchschnittlich übliche, notfallmässig abgegebene Menge. Zum anderen fanden sich auch Arzneimittel auf der Bestellung, die offensichtlich nicht notfallmässig oder im Rahmen der Direktversorung durch einen Psychiater abgegeben werden, wie Appetitzügler, Abführmittel, Tropfen gegen Haarausfall, Tabletten zur Senkung der Blutfettwerte, gegen Nikotinabhängigkeit und gegen Erektionsstörungen ${ }^{109}$. Das Bundesgericht bestätigte, dass es der fachtechnisch verantwortlichen Person hätte auffallen müssen, dass Art und Umfang der Bestellungen nicht mit einer Bewilligung eines Psychiaters zur Notfall- und Direktversorgung übereinstimmen kann. Insbesondere verwarf es das Argument der beschwerdeführenden fachtechnisch verantwortlichen Person, wonach ihr die persönliche Kontrolle der Medikamentenlieferung nicht zumutbar gewesen sein soll. Letztere hat die Bestellungen weder selbst kontrolliert noch durch entsprechende Weisungen sichergestellt, dass ihr zumindest die umfang-

$109 \overline{\text { BGer-Urteil 6B_444/2010 vom 16.9.2010, E. }}$ 4.1.2; Ursula Eggenberger Stöckli, Compliance im Pharmabereich, in: Newsletter 2/2012, hrsg. von Bratschi Wiederkehr \& Buob, S. 2 f. (nachfolgend: Eggenberger Stöckli, 2012). reichen, häufigen oder auffälligen Aufträge bzw. jene aus einem Gebiet mit einem grundsätzlichen Abgabeverbot zur Kontrolle vorgelegt wurden. Das Bundesgericht resümierte, dass die Beschwerdeführerin als studierte Apothekerin über das notwendige Wissen verfügt, pro Arztkategorie interne Kontrollmechanismen einzuführen, die die Aufdeckung kritischer Bestellungen erlauben $^{110}$. Entsprechend bestätigte es die Verurteilung der fachtechnisch verantwortlichen Person wegen Widerhandlung gegen das Heilmittelgesetz.

33 Dieser Fall zeigt eindrücklich, dass die Gerichte an das Pflichtbewusstsein der fachtechnisch verantwortlichen Person hohe Anforderungen stellen, was sich mit Blick auf die potentielle Gesundheitsgefährdung der Bevölkerung rechtfertigt ${ }^{111}$. Für die betroffene fachtechnisch verantwortliche Person bedeutet dies ein erhebliches Strafverfolgungsrisiko. Diese Rechtsprechung hat denn auch für $\mathrm{F}$ zur Folge, dass er mit dem Argument, nicht er selbst, sondern sein Mitarbeiter habe die Grosshandelstätigkeit der X AG genehmigt, einer Strafe nicht entgehen kann. Dies zumindest dann nicht, wenn er nicht darlegen kann, dass er umfassende Vorkehrungen getroffen und griffige Kontrollmechanismen eingeführt hat, um Verstösse gegen das Heilmittelgesetz durch seine Untergebenen $\mathrm{zu}$ verhindern und abzuwenden ${ }^{112}$.

$110 \overline{\text { BGer-Urteil 6B_444/2010 vom 16.9.2010, E. 7.2 }}$ vgl. auch Eggenberger Stöckli, 2012, S. 2; www.swissblawg.ch/2010/10/6b4442010widerhandlung-gegen-das-hmg.html.

111 So auch Eggenberger Stöckli, 2012, S. 3.

112 Vgl. auch Gfeller (Fn. 78), S. 274. 
34 Schliesslich wird der Vorgesetzte auch dann bestraft, wenn er fahrlässig zulässt, dass ihm hierarchisch Untergeordnete gegen die Heilmittelvorschriften verstossen $^{113}$. Kein strafrechtlicher Vorwurf kann der fachtechnisch verantwortlichen Person hingegen dann gemacht werden, wenn sie um die regelwidrigen Aktivitäten der ihr untergebenen Person weder wissen musste noch bei Aufwendung aller Sorgfalt solche hätte annehmen müssen ${ }^{114}$. Allerdings liegt die Schwelle für das «Annehmenmüssen» von Gesetzesverstössen eines Untergeordneten tief: Für die Strafbarkeit des Vorgesetzten reicht es bereits aus, "wenn die Gefahr der Begehung einer Anlasstat objektiv betrachtet betriebs- und branchentypisch ist»115. Weil die Pharmabranche durch das Heilmittelgesetz und seine Ausführungsverordnungen stark reguliert ist, dürfte bei denjenigen Personen, die direkt mit dem Ein- und Verkauf bzw. der Herstellung der Arzneimittel beschäftigt sind, das Risiko einer Anlasstat bzw. eines Verstosses gegen die heilmittelrechtlichen Vorschriften und damit das Risiko einer Haftung des Vorgesetzten grösser sein als bei Personen, die nur indirekt mit Arzneimitteln zu tun haben, wie das z.B. in der Administration oder der Buchhaltung der Fall ist.

$113 \overline{\text { Eicker/Frank/Achermann (Fn. 35), S. 54; Oertle }}$ (Fn. 100), S. 141 ff. Die Strafbarkeit des Untergeordneten stellt damit nur eine objektive Strafbarkeitsbedingung dar (Eicker/Frank/Achermann (Fn. 35), S. 54; Gfeller (Fn. 78), S. 269; Hauri (Fn. 93), Art. 6 N 14c).

114 Eicker/Frank/Achermann (Fn. 35), S. 54; Oertle (Fn. 100), S. $189 \mathrm{ff}$.

115 Eicker/Frank/Achermann (Fn. 35), S. 54; vgl. auch Gfeller (Fn. 78), S. 268 f.; Oertle (Fn. 100), S. $106 \mathrm{ff}$.
35 In diesem Zusammenhang stellt sich die Frage, wie es sich mit der strafrechtlichen Verantwortlichkeit des Vorgesetzten der fachtechnisch verantwortlichen Person verhält. In kleineren Unternehmen können das die Geschäftsführer sein, in grösseren z.B. der Leiter der Compliance Abteilung. Der fachtechnisch Verantwortliche nimmt eine Sonderstellung innerhalb des Unternehmens ein: Er ist weisungsbefugt und in der Regel von der Geschäfts- und Produktionsleitung unabhängig. Durch die SwissmedicBewilligung wird die Sicherstellung der öffentlichen Gesundheit in einem bestimmten und abgesteckten Bereich dem Unternehmen bzw. der fachtechnisch verantwortlichen Person überbunden. Diese Aufgabe verlangt entsprechendes Fachwissen, welches der Vorgesetzte des fachtechnisch Verantwortlichen gerade in kleineren Unternehmen in der Regel nicht hat. Eine fachliche Kontrolle und Aufsicht scheidet in solchen Konstellationen aus. Allerdings kann der Vorgesetzte dann strafrechtlich verantwortlich gemacht werden, wenn er in Verletzung der gebotenen Sorgfalt z.B. zulässt, dass die fachtechnisch verantwortliche Person nicht regelmässig zur Arbeit erscheint, ihre Stellvertretung nicht (hinreichend) geregelt hat oder der Betriebsablauf durch die Untätigkeit des fachtechnisch Verantwortlichen gestört ist. Wie es sich darüber hinaus mit der Strafbarkeit des Vorgesetzten verhält, muss an dieser Stelle offen bleiben.

36 Zusammenfassend lässt sich festhalten, dass die Geschäftsherrenhaftung nach Art. 6 Abs. 2 und 3 VStrR für die fachtechnisch verantwortliche Person zu einer «Ausdehnung» ihrer strafrechtlichen Verantwortlichkeit führt. 


\section{Fazit}

37 Die vorstehenden Ausführungen haben aufgezeigt, dass die fachtechnisch verantwortliche Person verwaltungsstrafrechtlich stark exponiert ist ${ }^{116}$. Insbesondere durch die ihr zukommende Garantenstellung wird ihre Strafbarkeit «ausgedehnt» und das Risiko einer Strafverfolgung erhöht. In der Praxis kommt es nicht selten vor, dass die fachtechnisch verantwortliche Person insbesondere von kleineren in der Herstellung oder im Handel tätigen Pharmabetrieben bei ihrer ersten Einvernahme überrascht reagiert, wenn ihr vom Untersuchungsleiter ihre Verantwortlichkeiten im Zusammenhang mit einem vermuteten Verstoss gegen die Heilmittelvorschriften vor $\mathrm{Au}-$ gen geführt werden. Für die Abwendung einer Verurteilung durch Swissmedic oder die kantonalen Gerichte ist es zu diesem Zeitpunkt zu spät. Die fachtechnisch verantwortliche Person muss sich daher vor Übernahme dieser Schlüsselrolle in einem in der Pharmabranche tätigen Unternehmen umfassende Klarheit über ihre Zuständigkeiten, Kompetenzen und Verantwortlichkeiten verschaffen - dies nicht zuletzt auch zu ihrem eigenen Schutz.

116 So auch Cordoba (Fn. 2), Rz 89, 109. 